\title{
CUBIC AND ULTIMATE GROUPS OF COMPLETE SYMMETRY
}

\author{
BY 1. S. ZHELUDEV* \\ (Department of Physics, Indian Institute of Science, Bangalore-12, India) \\ Received October 15, 1963 \\ (Communicated by Dr. R. S. Krishnan, F.A.sc.)

\section{INTRODUCTION}

THE idea of complete symmetry of tensors was introduced in our previous papers. The groups of complete symmetry of polar and axial tensors of the second rank have been described earlier (Zheludev, $1960 \mathrm{a}$ ). Ultimate groups of complete symmetry for these tensors were also described (Zheludev, $1960 \mathrm{~b}$ ). All crystallographical groups of complete symmetry and the changes of complete symmetry during ferroelectric and ferromagnetic transitions in the crystals were presented in a subsequent paper (Zhelidev, 1964). The relations between antisymmetry, magnetic symmetry and complete symmetry are given in the paper by Zheludev $(1960 \mathrm{c}$ ). Cubic groups of crystal symmetry will be determined in detail in this paper [in the paper (Zheludev, loc. cit.) they were presented without a conclusion]. Ultimate groups of complete symmetry of finite figures and the relation between complete symmetry and physical properties of the crystals are also discussed here.

\section{Cubic Groups of Complete Symmetry}

As it was shown earlier (Zheludev, 1957), crystallographical groups of usual symmetry can be obtained as a result of scalar (including pseudoscalar) and vector (including pseudovector) actions on the elementary parallelepiped of the cubic system. The groups of symmetry, in this case, are found as a result of superposition of the elements of the symmetry of the original elementary cubic parallelepiped (cube) and external action. Within the limits of usual symmetry, it is possible to establish five above-mentioned sets of values differing in symmetry and resulting in the groups: $\mathrm{m} 3 \mathrm{~m}(\overline{6} / 4) \dagger$

\footnotetext{
* Visiting Professor from the Institute of Crystallography, U.S.S.R. Academy of Science, Moscow, U.S.S.R.

+ The symbols of the groups of usual and complete symmetry assumed in the international convention are given. The notations by Shubnikov (see Zheludev, 1964) are given in brackets.
} 
scalar action $432(3 / 4)$-pseudoscalar action, $\overline{43} \mathrm{~m}(3 / \overline{4})$-vector action, $\mathrm{m} 3(\overline{6} / 2)$-pseudovector action, as well as in the group $23(3 / 2)$ presenting combinations of the above actions. The original elementary parallelepiped in this case should be considered as a coloured cube. This parallelepiped is equal to the grey (not painted) parallelepiped subjected to the scalar action [group $\mathrm{m} 3 \mathrm{~m}(\overline{6} / 4)$ ].

From the point of view of complete symmetry, it is possible to consider cubic groups of crystal symmetry in a more detailed manner, since in this case, one succeeds in ascribing different symmetry to the broader class of the combinations of actions than it is possible in the case of usual symmetry. In this case, the initial elementary parallelepiped is considered to be a " grey" (not coloured) cube. Such a cube, by definition, possesses all elements of symmetry of the figure belonging to any cubic group: three axes $4, \overline{4}, \overline{4}$ and $\underline{4}$, four axes $6, \overline{6}, \overline{6}$, six axes $2, \overline{2}, \overline{2}, \underline{2}$, nine planes and nine antiplanes of symmetry, the centre of symmetry and anticentre of symmetry. From all external actions, it is those groups that have the symmetry of cubic groups that will be considered here. Such groups of symmetry possess more than one operation of rotation around the axis having the order higher than the second one. It is easy to see that scalar effect (symmetry $\infty / \mathrm{m} . \infty: \mathrm{m}$ ), pseuodoscalar effect (symmetry $\infty / \mathrm{m} . \infty: \underline{m}$.) and a combination of these effects (symmetry $\infty / \infty: 2$ ) satisfy this condition. Besides, the set of four polar vectors directed perpendicularly to the edges of a cubic tetrahedron, the same set of axial vectors and any combination composed of the two latter effects in a combination with scalar and pseudoscalar effects comply with the above-said condition.

Cubic groups of complete symmetry will be found in this case, by considering the superposition of the elements of complete symmetry using generalized Curie principle of symmetry (Zheludev, 1964). It is obvious that cubic groups will be obtained in case of scalar and pseudoscalar action during any mutual orientation of a "grey" cube and actions. The group $\mathrm{m} 3 \mathrm{~m} .(\overline{6} / 4)$ will answer for scalar action, while the group $\mathrm{m}^{\prime} 3 \mathrm{~m}^{\prime}(\overline{6} / 4)$ (a new group as compared with usual symmetry) will satisfy the pseudoscalar action. The group $432(3 / 4)$ will satisfy the combination of scalar and pseudoscalar actions. In the case of vector and pseudovector actions (above-mentioned sets of four vectors or pseudovectors), cubic groups can be obtained only when the vectors are oriented along the space diagonals of the cube 
In the case of polar vectors, a superposition of the elements of symmetry of the action with the elements of symmetry of "a grey" cube results in a group $\mathrm{m}^{\prime} 3 \mathrm{~m}(\overline{6} / 4)$. In case of axial vectors it results in the group $\mathrm{m} 3 \mathrm{~m}^{\prime}$ $(\overline{0} / 4)$.

Cubic groups can also be obtained in the case of various (double, triple and four-fold) combinations of scalar, pseudoscalar, vector and pseudovector effects. It appears that there are 11 cubic groups which differ in complete symmetry (Fig. 1). One of these groups $23(3 / 2)$, the least symmetrical one, (as is seen from the figure) can be presented by five different combinations of the effects (four triple combinations of the type-scalar-vector, pseudovector; pseudo-scalar-vector, pseudo-vector, etc.) and one combination including all types of the effects (scalar, pseudoscalar, vector and pseudovector). The rest of the groups of complete symmetry (except the group 23) have only one interpretation with the help of actions.

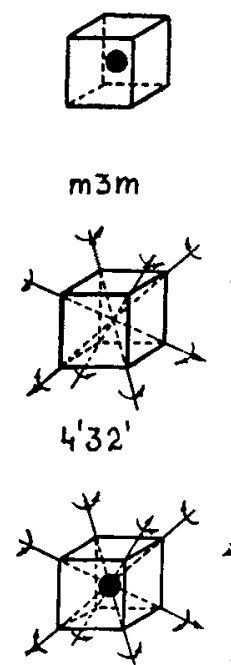

23

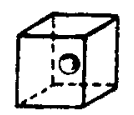

$m^{\prime} 3 m^{\prime}$

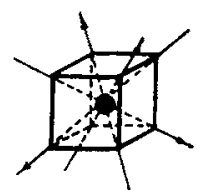

$\overline{4} 3 \mathrm{~m}$

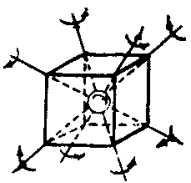

$2^{2}$

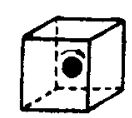

432

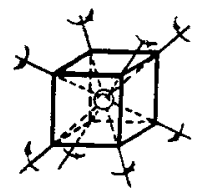

$\overline{4}^{\prime} 3 \mathrm{~m}^{\prime}$

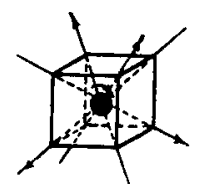

23

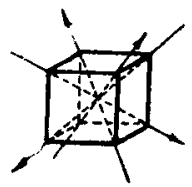

$m^{\prime} 3 m$

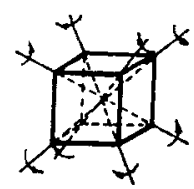

$m 3 m^{\prime}$

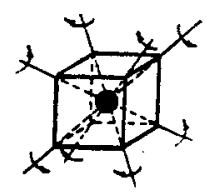

$m 3$

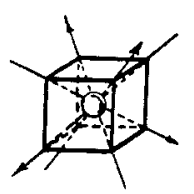

$m^{\prime} 3$

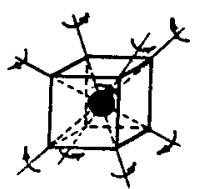

23

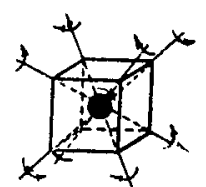

23

Fig. 1. The interpretation of cubic groups of complete symmetry is attained by means of scalar, pseudoscalar, vector and pseuodovecor actions.

It is the cases of scalar and pseudoscalar effects of one sign that are considered in the figures presented in Fig. 1. At the same time, for instance, another presentation is conceivable for the group $m 3 \mathrm{~m}(\overline{6} / 4)$. In this case, the scalar effect will be a "negative" one (hydrostatic compression in particular) in contrast to a "positive" effect which is shown conventionally by 
a black circle. In its turn, the figure presenting the group $\mathrm{m}^{\prime} 3 \mathrm{~m}^{\prime}(\overline{6} / 4)$ can be considered as the "right" one, while the "left" figure belonging to this group which can be conventionally designated by a circle with an arrow directed to the left is also conceivable, etc. All such cases that do not result in new groups of complete symmetry are not considered in Fig. 1.

We do not know of the other types of effects (except those shown in Fig. 1) which would also result in cubic groups. It is therefore, possible to assert that there are only 11 cubic crystallographical groups of complete symmetry. All of them can be considered to te a result of external actions (described by tensors of the second rank) on the "grey" cube. Practical advisability of tensor interpretation of the groups of complete symmetry of the crystals will be considered below.

The set of the elements of symmetry of the figures belonging to cubic crystallographical groups is given in Table 1 . The axes of symmetry are

TABLE I

Cubic groups of complete symmetry

\begin{tabular}{|c|c|c|c|c|}
\hline No. & $\begin{array}{l}\text { Notation } \\
\text { of the } \\
\text { classes by } \\
\text { Shubnikov }\end{array}$ & $\begin{array}{l}\text { Inter- } \\
\text { national } \\
\text { notations } \\
\text { of the } \\
\text { classes }\end{array}$ & $\begin{array}{l}\text { The formula } \\
\text { of complete } \\
\text { symmetry }\end{array}$ & $\begin{array}{l}\text { Subclass } \\
\text { of usual } \\
\text { symmetry }\end{array}$ \\
\hline 1 & $3 / 2$ & 23 & $3 \mathrm{~L}^{2} \underline{4} \mathrm{~L}^{3}$ & $3 / 2$ \\
\hline 2 & $3 / \underline{4}$ & $4^{\prime} 3 m^{\prime}$ & $3 \mathrm{~L}_{\underline{\underline{I}}}{ }^{2} 4 \mathrm{~L}^{3} 6 \mathrm{P}$ & $3 / 2$ \\
\hline 3 & $3 / \overline{4}$ & $\overline{4} 3 \mathrm{~m}$ & $3 \mathrm{~L}_{\pi}^{2} 4 \mathrm{~L}^{3} 6 \mathrm{P}$ & $3 / 4$ \\
\hline 4 & $\underline{6} / 2$ & $\mathrm{~m}^{\prime} 3$ & $3 \mathrm{~L}_{\underline{\underline{z}}}^{-2} 4 \mathrm{~L}_{\underline{\delta}}^{3} 3 \underline{\mathrm{PC}}$ & $3 / 2$ \\
\hline 5 & $6 / 2$ & $\mathrm{~m} 3$ & $3 \mathrm{~L}_{\overline{2}}{ }^{2} 4 \mathrm{~L}_{\overline{6}}{ }^{3} 3 \mathrm{PC}$ & $6 / 2$ \\
\hline 6 & $3 / \underline{4}$ & $4^{\prime} 32^{\prime}$ & $3 \mathrm{~L}_{\underline{4}}{ }^{2} 4 \mathrm{~L}^{3} 6 \mathrm{~L}_{2}$ & $3 / 2$ \\
\hline 7 & $3 / 4$ & 432 & $3 L^{4} 4 L^{3} 6 L^{2}$ & $3 / 4$ \\
\hline 8 & $\overline{6} / 4$ & $\mathrm{~m}^{\prime} 3 \mathrm{~m}$ & $3 \mathrm{~L}^{2}{ }_{4} \bar{Z}^{-4 \mathrm{~L}_{5}}-\mathrm{L}_{2} 6 \mathrm{P} 3 \underline{\mathrm{PC}}$ & $3 / 4$ \\
\hline 9 & $6 / 4$ & $\mathrm{~m}^{3} \mathrm{~m}^{\prime}$ & $3 \mathrm{~L}_{\underline{4}-\pi^{2}} 4 \mathrm{~L}_{6}{ }^{3} 6 \mathrm{~L}_{2}{ }^{2} 6 \mathrm{P} 3 \mathrm{PC}$ & $\overline{6} / 2$ \\
\hline 10 & $\underline{6} / 4$ & $m^{\prime} 3 m^{\prime}$ & $3 \mathrm{~L}_{\underline{\Phi}} 4 \mathrm{~L}_{\tilde{E}}{ }^{3} 6 \mathrm{~L}_{\underline{\underline{E}}}{ }^{29} \underline{\underline{\mathrm{PC}}}$ & $3 / 4$ \\
\hline 11 & $\overrightarrow{6} / 4$ & $\mathrm{~m} 3 \mathrm{~m}$ & $3 \mathrm{~L}_{\overline{4}}{ }^{4} 4 \mathrm{~L}_{5}^{-3} 6 \mathrm{~L}_{\overline{2}}^{2} 9 \mathrm{PC}$ & $6 / 4$ \\
\hline
\end{tabular}


designated by the letters $L$ indicating the order of common axes on the top and the order of the antirotation, mirror and antimirror axes below. The plane of symmetry is designated by the letter $P$, an antiplane is designated by $\underline{P}$, the centre of symmetry is designated by $\mathrm{C}$ and an anticentre by $\underline{C}$.

\section{Ultimate Groups of COMPLETE SYMMETRY of Finite Figures}

Ultimate groups are those that descrite the symmetry of the figures that have symmetry axes of infinite orcier $(\infty)$ (sphere, cylinder, cone, etc.).

As was shown earlier (Zheludev, $1960 \mathrm{~b}$ ) there are 14 ultimate groups of complete symmetry for polar and axial tensors of the second rank. Ultimate groups for finite figures can be obtained by arranging into rows crystallographical groups having identical type of symmetry. 79 crystallographical groups of lower and medilm system can be arranged into 24 such rows (Zheludev, 1964). 11 out of these rows end in ultimate groups of complete symmetry of tensors of the second rank. These rows are as follows:

$$
\begin{array}{lll}
\mathrm{m} .1: \mathrm{m}, \mathrm{m} \cdot 2: \mathrm{m}, \mathrm{m} \cdot 3: \mathrm{m}, & . & \mathrm{m} \cdot \infty: \mathrm{m} \\
1 . \mathrm{m}, 2 . \mathrm{m}, 3 \cdot \mathrm{m} & \ldots & \infty . \mathrm{m} \\
1: \underline{2}, 2: \underline{2}, 3: \underline{2} & \ldots & \infty: \underline{2}
\end{array}
$$

13 other rows

$$
\begin{aligned}
& \underline{2}: \underline{\mathrm{m}} ; \underline{4}: \underline{\mathrm{m}} \\
& 2 . \mathrm{m} ; \underline{4} . \mathrm{m} \\
& 2: 2 ; \underline{4}: 2
\end{aligned}
$$

do not end in ultimate groups of complete symmetry of tensors of the second rank, and, generally speaking, they cannot end in ultimate groups in the sense of this word, since they do not result in the groups that have simple axes of symmetry of infinite order $(\infty)$. At the same time, it is possible to introduce the idea about ultimate groups for such rows. In this case, ultimate groups will be of conventional character, since they cannot be presented as finite figures by means of spheres, cones and cylinders. The notations of the axes of symmetry $\bar{\infty}, \bar{\infty}, \infty$ in these conventional ultimate groups do 
not indicate that they are present in some ultimate figures but point to the fact that when proper rows are continued, the figures presenting inem can have the axes $\overline{\mathbf{N}}, \bar{N}, N$ (where $N$ is the order of an axis) of any high order. Some of the latter rows have the form

$$
\begin{aligned}
& \text { 2.m; 4.m;6.m... } \\
& \underline{2} . \mathrm{m} ; \underline{\overline{4}} \cdot \mathrm{m} ; \overline{\mathbf{6}} \cdot \mathrm{m} . . . \bar{\infty} \cdot \mathrm{m}
\end{aligned}
$$

Three out of the ultimate groups of symmetry of tensors of the second rank, namely, the groups $\infty / \mathrm{m} . \infty: \mathrm{m}, \infty / \underline{\mathrm{m}} \cdot \infty: \underline{\mathrm{m}}$ and $\infty / \infty: 2$ can be considered as the ultimate ones for the cubic groups $\overline{6} / 4, \overline{6} / 4$ and $3 / 4$ respectively. However, this "limit" is not so obvious as in the rows of finite figures, between above cubic and ultimate groups there is only one figure possessing corresponding type of symmetry

$$
\begin{array}{ll}
\overline{6} / 4 ; 3 / \overline{10} ; & \infty / \mathrm{m} . \infty: \mathrm{m} \\
\overline{6} / 4 ; 3 / \overline{10} ; & \infty / \mathrm{m} . \infty: \mathrm{m} \\
3 / 4 ; 3 / 5 ; & \infty / \infty: 2 .
\end{array}
$$

The group 3/10 can be obtained as a result of scalar action on "grey" icosahedron. The group $3 / \overline{10}$ is obtained as a result of pseudoscalar action, and group $3 / 5$ is a result of combined (scalar and pseudoscalar) action on the icosahedron.

For the remaining eight cubic groups it is also possible to introduce the idea about conventional ultimate groups. Note that in this case, there are no groups at all having the same type of symmetry tetween cubic groups and ultimate groups. When writing the symbols of conventional ultimate groups, the symmetry of the diameters of "ultimate" spheres corresponding to the symmetry of the directions in the cubes representing one or another group of symmetry $(\underline{6} / \underline{4} \rightarrow \infty / \mathrm{m} \cdot \infty: \underline{\mathrm{m}}, 3 / \underline{4} \rightarrow \infty / \infty: \underline{2}$, etc.,) will be shown.

Thus, together with the 21 conventional ultimate groups introduced, 35 ultimate groups of complete symmetry of finite figures are considered here. These are as follows: $\infty / \mathrm{m} \cdot \infty: \mathrm{m} ; \infty / \underline{\mathrm{m}} \cdot \infty: \mathrm{m} ; \infty / \infty: 2 ; \mathrm{m} . \infty: \mathrm{m}$; m. $\infty: \underline{\mathrm{m}} ; \underline{\mathrm{m}} . \infty: \mathrm{m} ; \mathrm{m} . \infty: \underline{\mathrm{m}} ; \infty: 2 ; \infty . \mathrm{m} ; \infty: \mathrm{m} ; \infty . \underline{\mathrm{m}} ; \infty: \underline{\mathrm{m}} ;$ 
$\infty: \underline{2} ; \infty ; \infty / \underline{\mathrm{m}} . \infty: \mathrm{m} ; \infty / \mathrm{m} . \infty: \underline{\mathrm{m}} ; \infty / \infty: \underline{2} ; \infty / \infty: \mathrm{m} ; \infty / \infty: \underline{\mathrm{m}} ;$ $\infty / \infty . \mathrm{m} ; \infty / \infty . \mathrm{m} ; \infty / \infty ; \mathrm{m} . \infty: \mathrm{m} ; \mathrm{m} . \infty: \underline{\mathrm{m}} ; \infty: \mathrm{m} ; \infty: \underline{\mathrm{m}} ; \infty . \mathrm{m}$;

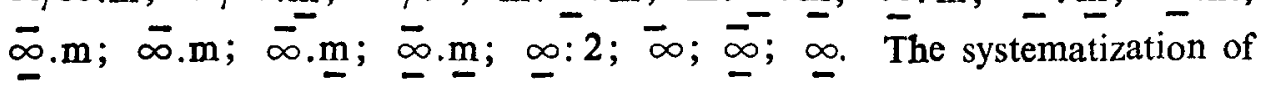
crystallographical groups according to their classification into one or another ultimate group is presented in Table II. Since some of the first terms in the rows represent the identical groups but are written down in a different manner, let us show the connection between the different notations

$$
\begin{array}{ll}
1 \cdot \underline{m}=\underline{1}=1: \underline{m}=\underline{m} & 1: 2:=\underline{2} \\
\underline{m} \cdot 1: \mathrm{m}=\mathrm{m} \cdot 1: \underline{m}=\underline{2} \cdot \mathrm{m} & \underline{\mathbf{2}} \cdot \mathrm{m}=\underline{2}: \mathrm{m} \\
\mathrm{m} \cdot \underline{2}: \underline{\mathrm{m}}=\underline{\mathrm{m}} \cdot 2: \mathrm{m} & \underline{2} \cdot \underline{\mathrm{m}}=2: \underline{\mathrm{m}} \\
\mathrm{m} \cdot 2: \underline{\mathrm{m}}=\mathrm{m} \cdot \underline{2}: \mathrm{m} & 1: 2=2 \\
\mathrm{~m} \cdot 1: \mathrm{m}=2 \cdot \mathrm{m} & 1 \cdot \mathrm{m}=1: \mathrm{m}=\mathrm{m} \\
\overline{2} \cdot \mathrm{m}=\underline{2}: \underline{\mathrm{m}} & \underline{\mathrm{m}} \cdot 1: \underline{\mathrm{m}}=2 \cdot \underline{\mathrm{m}} \\
\underline{2}: 2=2: \underline{2} & \overline{2} \cdot \mathrm{m}=2: \mathrm{m} .
\end{array}
$$

Family tree of ultimate groups of complete symmetry is given in Fig. 2.

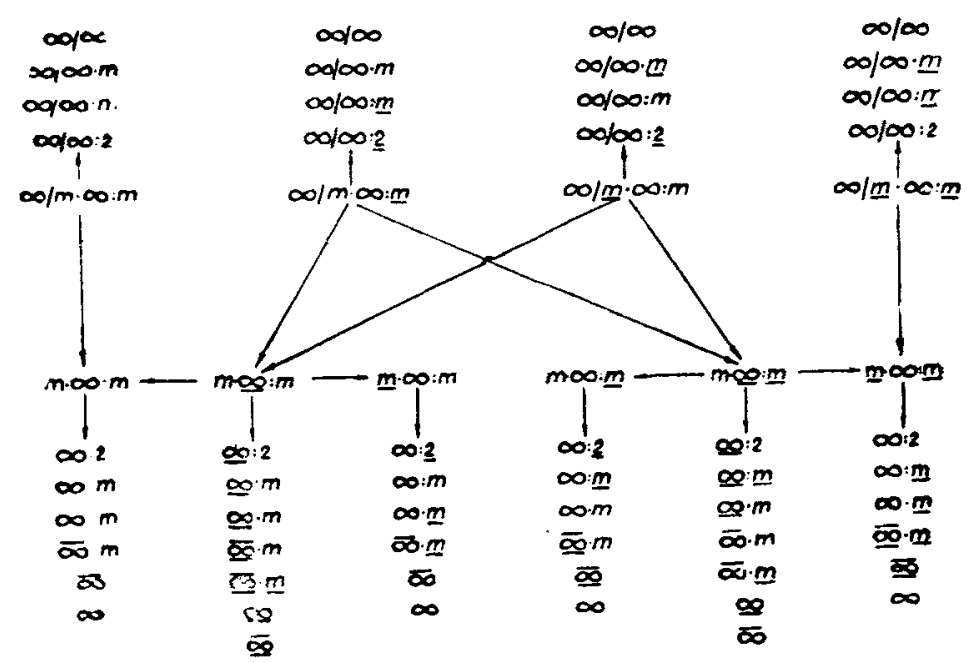

Fic. 2. Family tree of ultimate groups of complete symmetry of finite figure: 


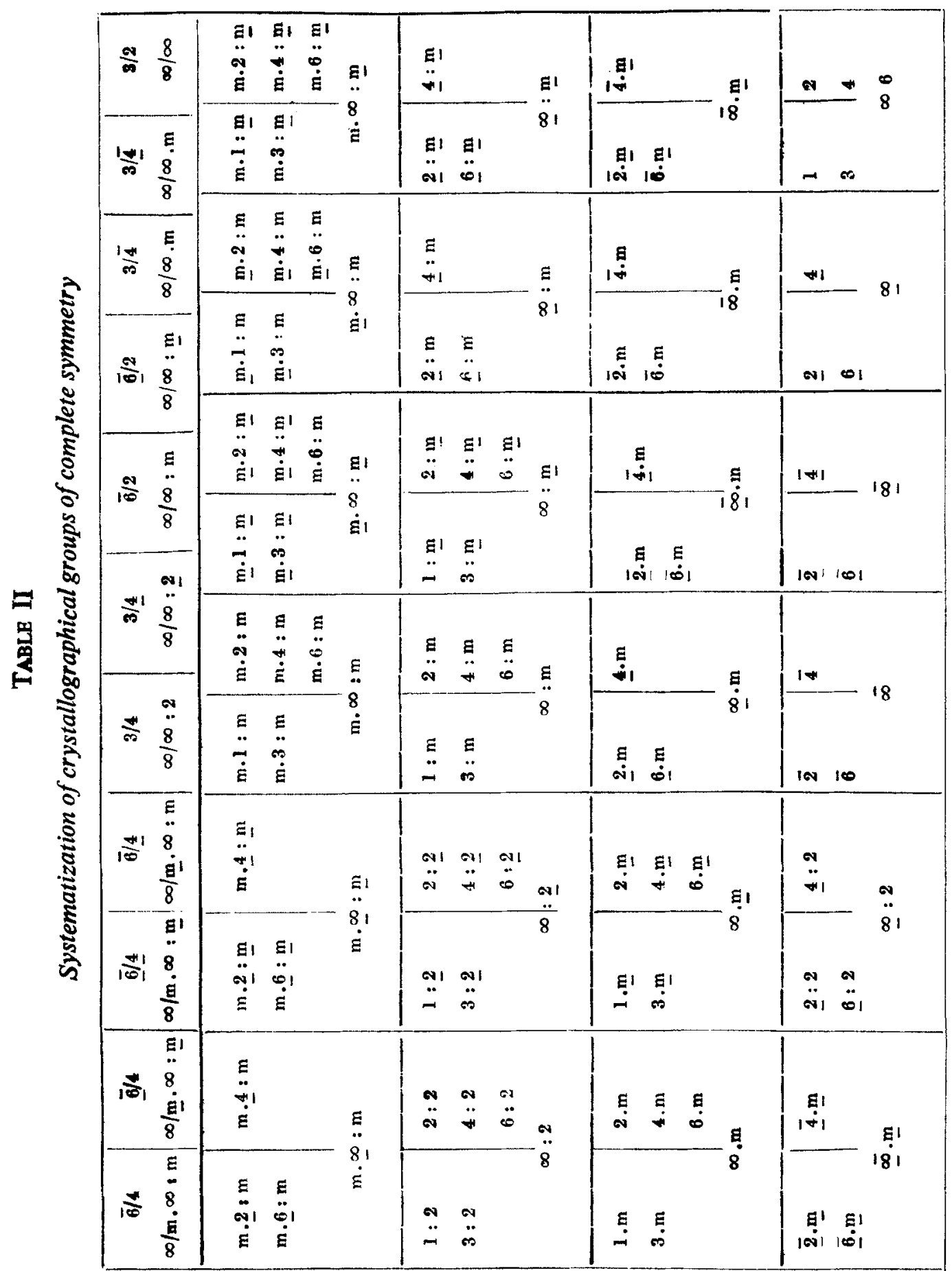




\section{Complete Symmetry and Physical Properties of the Crystals}

A geometrical interpretation of the cubic groups of complete symmetry by means of scalar and vector actions gives an obvious opportunity for discussing the physical properties of the crystals. For instance, it is seen from Fig. 1 that an optical activity is possible in cubic crystals of the classes $\underline{6} / 4$; $3 / 4 ; 3 / 4 ; 6 / 2 ; 3 / 2$. This is determined by the presence of pseudoscalar actions in the figures representing these classes. There are polar directions in the crystals of the classes $\underline{6} / 4 ; 3 / 4 ; 3 / 4 ; \underline{6} / 2$ and $3 / 2$. This means that piezoeffect is possible in them. The presence of polar directions corresponds to the presence of polar vector components in the actions presenting the symmetry of the listed classes, etc.

Scalar (pseudoscalar) and vector (pseudovector) components are shown as main components in Fig. 2. The subjected groups in which there exists one or another action are also given. While using Table II and Fig. 2 it is possible to select the groups containing one or another main action without using geometrical interpretation. So, it is possible to find out from Fig. 2 and Table II that there are axial directions in the crystals of the classes $\overline{6} / \underline{4} ; 3 / \underline{4} ; 3 / \underline{4} ; \overline{6} / 2 ; 3 / 2$, etc.

Geometrical interpretation of all groups of complete symmetry appears to be rather extensive. However, the most essential results can be obtained considering only the most important groups. First of all these are polartensor groups, subjected to the group $\mathrm{m} . \infty: \mathrm{m}$. The most highly symmetrical groups subjected to the above group are crystallographcial groups m.6:m and $\mathrm{m} .4: \mathrm{m}$. All groups of usual symmetry (except cubic group) are subjected to these groups. Hexagonal and tetragonal prisms coloured into certain colour give the symmetry of the crystals belonging to these groups. One colour of the figures representing these groups in complete symmetry allows us to consider the groups of usual symmetry as polar ones by the sign $(+$ or -$)$. It is easy to see by analogy that the groups $\underline{m} .6: \underline{m}$ and $\underline{m}, 4: \underline{m}$ can be presented as prisms possessing a certain sign of the enantiomorphism (the crystal group polar by the sign of enantiomorphism, i.e., either right or left ones). The presence of axial-tensor components in the actions is characteristic for all groups representing the latter groups.

The groups subjected to the group $\mathrm{m} . \infty \mathrm{m}$, are of two kinds. In its turn, each of them can be interpreted in two different ways. For instance, 
the group $\mathrm{m} .4: \mathrm{m}$ can be interpreted by means of polar tensors (Fig. $3 a, b$ ) and by means of axial vectors (Fig. $3 c$ ). The group m.6: $\mathrm{m}$ can be presented by means of axial tensors (Fig. $3 d$ ) and by means of polar vectors (Fig. $3 e, f$ ). The same is the situation during the interpretation of the groups subjected to the group m. $\infty: m$ (Fig. 4). In connection with the possible double interpretation of the groups in question $\mathrm{m} . \infty: \mathrm{m}$ and $\mathrm{m} . \infty: \mathrm{m}$, crystallographical groups are divided into two parts (where it is necessary) in Table II. For instance, the groups including vector and axial-tensor components in the row $\bar{\infty} . \mathrm{m}$ have the axes $\overline{4}$ and those including pseudovector and polar-tensor components have the axes $\overline{2}$ and $\overline{\boldsymbol{\sigma}}$, etc.
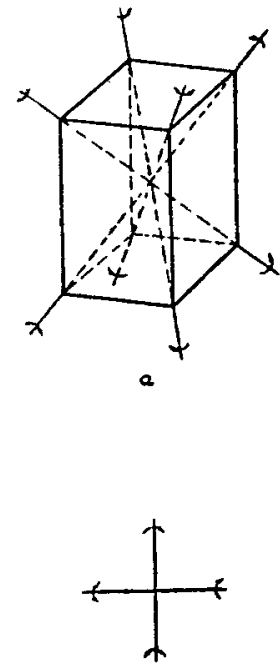

6

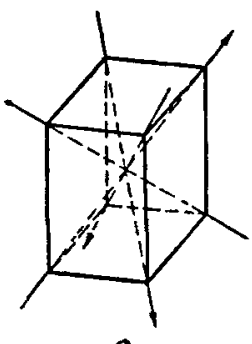

c

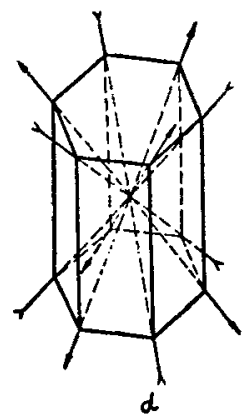

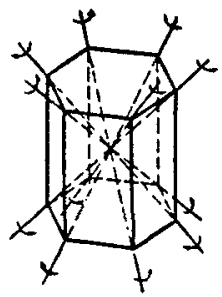

6

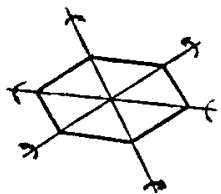

$f$

Fic. 3. Geometrical interpretation of crystallographical groups subjected to the grou $\mathrm{m} . \infty: \mathrm{m}$. $(a, b)$ Group $\mathrm{m} \cdot 4: \mathrm{m}$. The interpretation is attained by means of polar tensor (space and plane variants). (c) Group m.4: m. The interpretation is attained by means of axial vectors. (d) group $\mathrm{m} .6: \mathrm{m}$. The interpretation is attained by means of axial tensors. $(e, f)$ Group m.6:m. The interpretation is attained by means of polar vectors (space and plane variants).

The use of Figs. 3, 4 and the Table allows to select the groups of complete symmetry describing the crystals of intermediate and lower systems possessing any properties. It follows frcm Table II and Fig. 2 that axial (pyromagnetic groups of complete symmetry (subjected to the group $\mathrm{m}, \infty: \mathrm{m}$ ) 


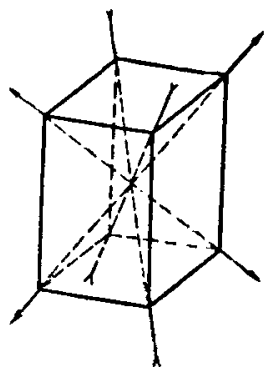

a

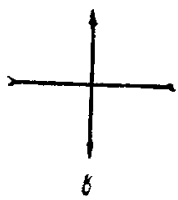

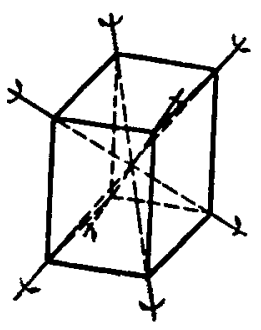

6

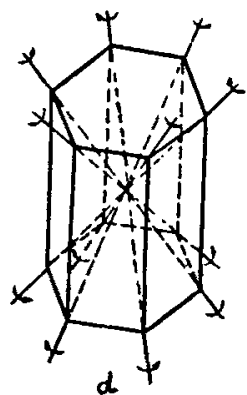

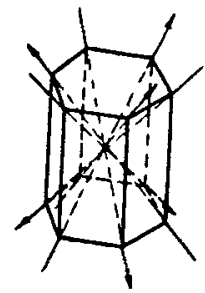

e

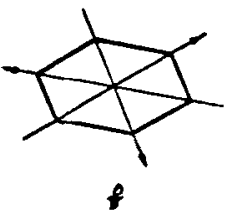

Fio. 4. Geometrical interpretation of the groups subjected to the group $\mathrm{m} . \infty: \underline{\mathrm{m}} .(a, b)$ Group $\mathrm{m} .4: \mathrm{m}$. The interpretation is attained by means of axial tensors (space and plane variants). (c) Group m. $\underline{4}: \underline{\mathrm{m}}$. The interpretation is attained by means of polar vectors. (d) Group $\mathrm{m} . \underline{\mathrm{K}}: \underline{\mathrm{m}}$. The interpretation is attained by means of polar tensors. $(f, f)$ Group $\mathrm{m} \cdot 6: \mathrm{m}$. The interpretation is attained by means of axial vectors (space and plane variants).

are 31 groups: $1 ; \overline{2} ; 2 ; 2 ; \mathrm{m} ; \mathrm{m} ; 22: \mathrm{m} ; 2: \mathrm{m} ; 2: 2 ; 2 . \mathrm{m} ; 2 . \mathrm{m}$;

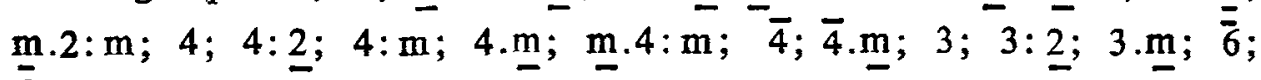
$\overline{6} \cdot \underline{\mathrm{m}} ; 3: \mathrm{m} ; \underline{\mathrm{m}} \cdot \overline{3}: \mathrm{m} ; 6 ; 6: \underline{2} ; \overline{6}: \mathrm{m} ; 6 \cdot \underline{\mathrm{m}} ; \overline{\mathrm{m}} \cdot 6: \mathrm{m}$. The crystals belonging to the groups subjected to the group $\infty: 2$ have directions which are both polar and axial ones. Hence, these crystals can have simultaneously both pyroelectric and pyromagnetic properties, etc.

Concluding the discussion about the connection between the complete symmetry and the physical properties of the crystals let us stress the remarkable fact that all cubic groups can te interpreted as a result of actions described only by polar and axial tensors of the second rank on the "grey" cube. In this connection it is possible to assume that any action in some point of space can be reduced to those mentiored here. Then, in the case of empty isotropic space, the action (or the system of acting forces) should be reduced only to those which are presented in Fig. 1. Apparently, when 
considering this action, it is possible, in particular, to have an idea about the symmetry of isolated fundamental particles.

\section{SUMmary}

It is shown that the systems of definite actions described by polar and axial tensors of the second rank and their combinations during the superposition of their elements of complete symmetry with the elements of complete symmetry of the "grey" cube, result in 11 cubic crystallographical groups of complete symmetry. There are 35 ultimate groups (i.e., the groups having the axes of symmetry of infinite order) in complete symmetry of finite figures. 14 out of these groups are ultimate groups of symmetry of polar and axial tensors of the second rank and 24 are new groups. All these 24 ultimate groups are conventional groups since they cannot be presented by certain finite figures possessing the axes of symmetry $\bar{\infty}, \infty, \infty$. Geometrical interpretation for some of the groups of complete symmetry is given. The connection between complete symmetry and physical properties of the crystals (electrical, magnetic and optical) is shown.

\section{ACKNOWLEDGEMENT}

The author of the paper is thankful to Professor C. V. Raman, Professor R. S、 Krishnan and Dr. P. S. Narayanan for the fruitful discussion of some points in this work and their help.

\section{REFERENCES}

1. Zheludev, I. S.

2.

3.

4.

5.

5.
.. Kristallografiya, 1957, 2, 728: English translation. Soviet Physics-Crystallography.

.. Kristallografiya, $1960 a, 5,346$ : English Translation. Soviet, Physics-Crystallography, 1960, 5, 328.

.. Kristallografiya, $1960 \mathrm{~b}, 5,508$ : English Translation. Soviet Physics-Crystallography, 1961, 5, 489.

.. Izvest. Akad. Nauk SSSR., Ser. Fiz. 1960 c, 24. 1436.

.. Proc. Ind. Acad. Sci. 196t, 59, 168. 